\title{
Calculation of a beam on an inhomogeneous basis
}

\author{
Nikolaj Atarov* \\ Moscow State University of Civil Engineering, Yaroslavskoe shosse, 26, Moscow, 129337, Russia
}

\begin{abstract}
The questions of analytical calculating the beam on the base with the modulus of deformation (modulus of elasticity), which is changed by thickness of the layer by power law, was deals in the article. The purpose of work was receiving finite expressions for reactive pressure of the base on a beam and internal efforts in a beam when using model of the base with two characteristics (coefficient of subgrade resistance). A system of differential equations second-order with respect to the displacements of points the surface of a layer with a continuously changing modulus of elasticity was obtained based on the minimum of the total potential strain energy. The calculation of the rigid beam on the base on the action of the symmetric load was performed and the formulas for the reactive pressures of the base were got. Numerical calculation is executed and the analysis of influence of change of the module of deformation of a layer by the amount of reactive pressure and the bending moment in a beam was given. It is shown that with increase in the module of deformation on layer thickness the base with two characteristics on nature of work is approaching vinklerovsky.
\end{abstract}

\section{Introduction}

In most engineering challenges, a soil ground, on which the footing of buildings, hydraulic structures, road and airfield pavements are based, is considered as an elastic foundation. As is known, the main difficulty in structural design on an elastic foundation is to determine the soil pressure to the structure. To do so, it is necessary to perform a schematization of the soil foundation and to make up its design diagram or model. Natural soils have a very wide variety of properties, in particular heterogeneity in the thickness of the foundation.

\section{Methods}

Let us consider an elastic layer of constant thickness underlain by an incompressible soil body and being under the vertical load (Fig. 1).

\footnotetext{
* Corresponding author: jet_15@list.ru
} 


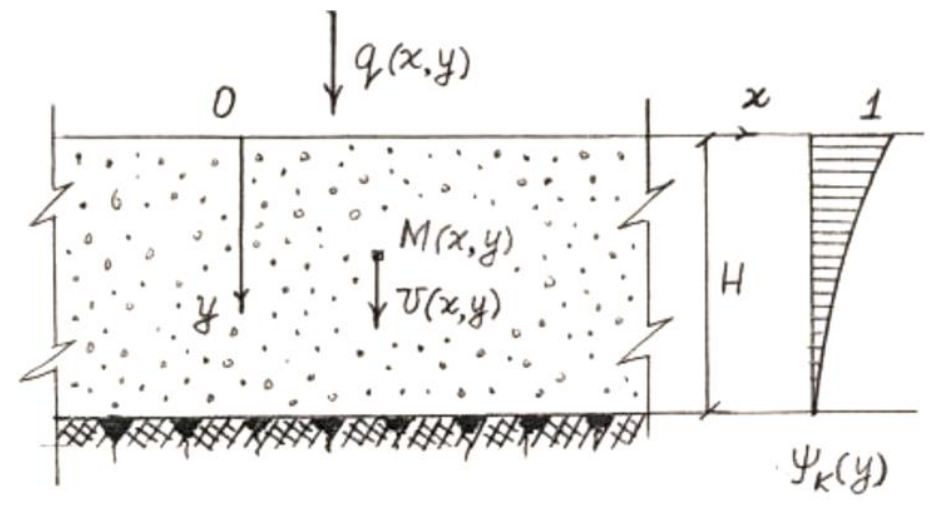

Fig.1. Elastic layer of soil. layer.

One can consider the soil own weight as the load acting on each particle inside the

As shown in works [1] and [2], the deformed state of the layer can be approximately determined using the Vlasov-Kantorovich variational method. Vertical node displacements of the layer under conditions of planar deformation are taken as a finite decomposition:

$$
v(x, y)=\sum_{k=1}^{m} V_{k}(x) \psi_{k}(y)
$$

In this expansion, the functions $V_{k}(x)$ are considered to be sought, and the functions $\psi_{k}(y)$ characterize the change in displacements along the thickness of the layer. They are taken in the form of some analytical expressions. The most convenient to take $\psi_{k}(0)=1$, $\psi_{k}(\mathrm{H})=0$. In this case, the functions $V_{k}(x)$ represent the node displacement (settlement) of the surface of the foundation. Horizontal node displacements of the layer can be set equal to zero.

With a relatively small thickness of the layer, the functions $\psi_{k}(y)$ can be assumed to vary linearly. For a layer of greater thickness or for a half-plane, the hyperbolic law of change of the functions $\psi_{k}(y)$ is applied.

Having formulated the expression for the total potential energy of deformation of the layer and having satisfied the condition of its minimum, we get system $n$ of second order ordinary differential equations with respect to the unknown functions $V_{k}(x)$ [1], [4]. The coefficients of this system express the resistance of the layer to compression and shear in integral form. They contain functions $\psi_{k}(y)$ and their derivatives.

When integrating the system of differential equations, $2 n$ constants appear, which are to be determined from the corresponding kinematic and static boundary conditions. To perform static boundary conditions, it is necessary to introduce generalized transverse forces characterizing the operation of shearing stresses $\tau_{y x}$ in the cross section $x$ on the possible displacements:

$$
S_{h}(x)=\int_{0}^{H} \tau_{y x} \cdot \psi_{k} d_{y}=\frac{E_{0}}{2\left(1+v_{0}\right)} \sum_{k=1}^{n} r_{k h} V_{k}^{\prime}(x),
$$

where $\quad r_{k h}=\int_{0}^{H} \psi_{k} \cdot \psi_{h} \cdot d y$

The $E_{0}$ and $v_{0}$ values are the modulus of elasticity and the Poisson's ratio of the material of the layer for planar deformation. 
Heterogeneity of the soil along the thickness of the layer can be taken into account in two ways.

The first method consists in splitting the soil ground into $\mathrm{n}$ layers with different elastic characteristics (Fig. 2).

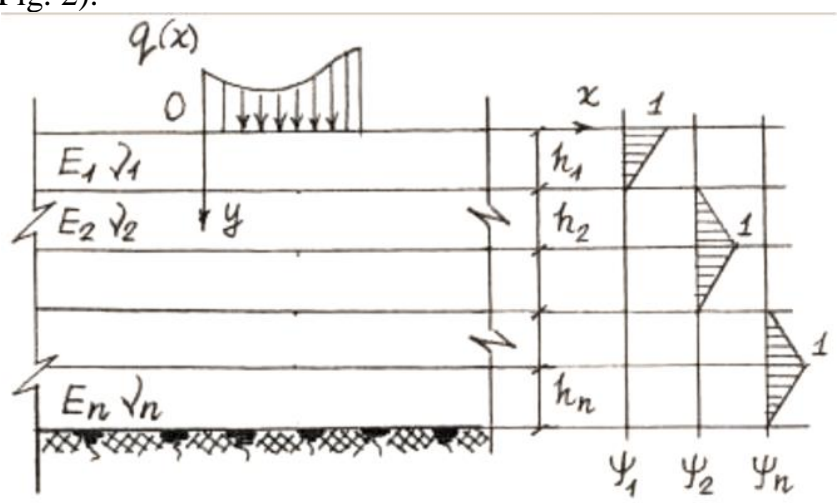

Fig.2. Multilayered base.

Within each layer, we can apply the linear law of change of functions $\psi_{k}(y)$ with values equal to one at the nodes of the surface of the layer and the boundaries of the layers. In this case, the required functions $V_{k}(x)$ are vertical node displacements.

In works [2] and [3], a matrix form of the solution of a system of differential equations describing the deformed state of the layer is given, and problems of beams design on a multi-layered foundation are considered. It is shown that for a homogeneous foundation with an increase in the number of conditional layers, the results of the beams design approach the solution data, using the elastic half-plane model [5].

The second method of taking into account the heterogeneity of the soil ground is to use the law of continuous variation of the elasticity modulus across the layer thickness. Professor G.K. Klein [6] offered to use a power law:

$$
E(y)=E_{n} \cdot y^{n},
$$

where $E_{n}$ is the value of the elastic modulus at a depth of $y=1$ with the dimension $\mathrm{kN} / \mathrm{cm}^{\mathrm{n}}$ +2 .

The graphs of the modulus of elasticity versus the parameter $n$ are shown in Fig. 3.

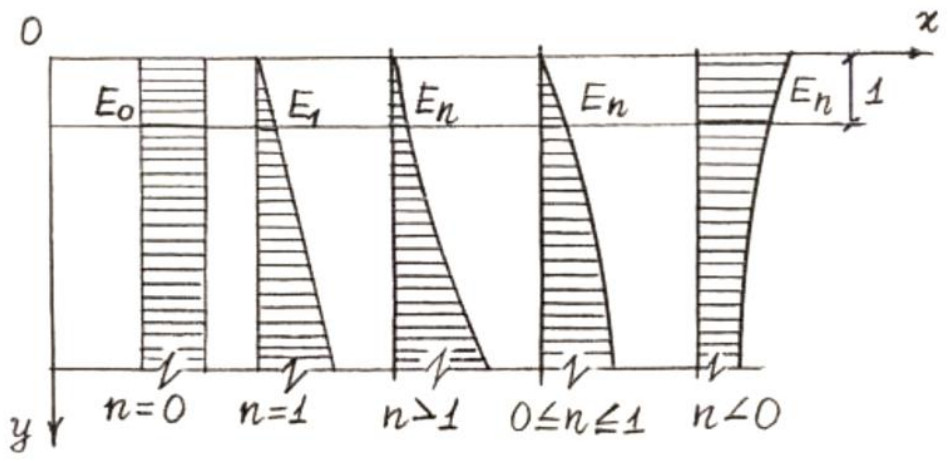

Fig.3. Change of the modulus of elasticity on the thickness of the layer.

In this case, under the action of the surface load, the system of differential equations describing the deformed state of the layer takes the following form: 


$$
\frac{1-v_{0}}{2} \sum_{k=1}^{n} r_{k h} \cdot V_{k}^{\prime \prime}-\sum_{k=1}^{n} s_{k h} \cdot V_{k}+\frac{1-v_{0}}{E_{n}} q_{h}=0,
$$

$h=1,2, \ldots \ldots, \mathrm{n}$, where

$$
r_{k h}=\int_{0}^{H} \psi_{k} \cdot \psi_{h} \cdot y^{n} d y, \quad s_{k h}=\int_{0}^{H} \psi_{k}^{\prime} \cdot \psi_{h}^{\prime} \cdot y^{n} d y, \quad q_{h}=\int_{0}^{H} q(x) \psi_{h} d y .
$$

Having expanded the (1) first member, we get the differential equation of the foundation model with two characteristics and with the modulus of elasticity varying according to the law (4):

$$
\begin{gathered}
2 t V_{1}^{\prime \prime}-k V_{1}+q_{1}=0, \\
\text { where } \quad t=\frac{E_{n}}{4\left(1-v_{0}\right)} \int_{0}^{H} \psi_{1}^{2} \cdot y^{n} d y, k=\frac{E_{n}}{1-v_{0}^{2}} \int_{0}^{H}\left(\psi_{1}^{\prime}\right)^{2} \cdot y^{n} d y
\end{gathered}
$$

The coefficient $k$ characterizes the resistance of the soil ground to compression and in this sense is analogous to the modulus of subgrade reaction in the Winkler model, and the coefficient $t$ is analogous to the shear.

To analyze the effect of the exponent $\mathrm{n}$ in formula (4), let us calculate a sufficiently rigid beam for the action of a symmetric load with a resultant $P_{0}$. The law of change of vertical displacements along depth we take as a linear one:

$$
\psi(y)=\frac{H-y}{H}
$$

For a sufficiently rigid beam, when determining the foundation pressure, one can ignore its bending and assume that at $-l \leq x \leq l \quad V_{1}=$ const. Outside the beam, the settlements of the foundation surface decrease exponentially as they move away from the ends (Fig. 4).

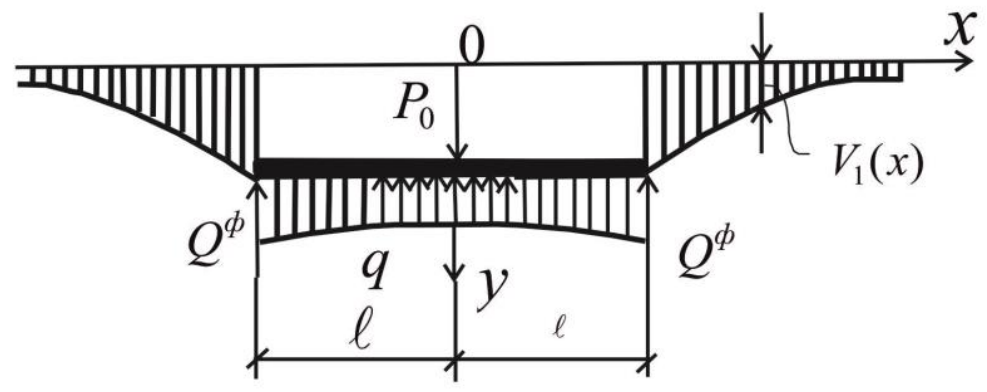

Fig.4. Reactive pressure of the base.

Having integrated the equation (7) and the boundary conditions at the ends of the beam, we get the following formulas for the foundation pressure:

$$
\begin{gathered}
q=\frac{P_{0}}{2 l} \frac{l \sqrt{\left(1-v_{0}\right)(n+2)(n+3)}}{l \sqrt{\left(1-v_{0}\right)(n+2)(n+3)}+H\left(1-v_{0}\right)}=\frac{P_{0}}{2 l} \bar{q} \\
Q^{\Phi}=\frac{P_{0}}{l} \cdot \frac{H\left(1-v_{0}\right)}{l \sqrt{\left(1-v_{0}\right)(n+2)(n+3)}+H\left(1-v_{0}\right)}=\frac{P_{0}}{2} \overline{Q^{\Phi}}
\end{gathered}
$$

where $Q^{\phi}$ - the so-called "fictitious" forces acting along the ends of the beam (Fig. 4), with the help of which the deformation of the foundation beyond its limits is taken into account. 


\section{Results}

Fig. 5 shows the dimensionless graphs of the variation of the foundation pressure $\bar{q}$ and $Q^{\phi}$ of the bending moment $\bar{M}$ in the middle of the beam under the action of a uniformly distributed load as a function of the parameter $n$. In the calculation, $H / l=1$ and $v_{0}=0,4$.

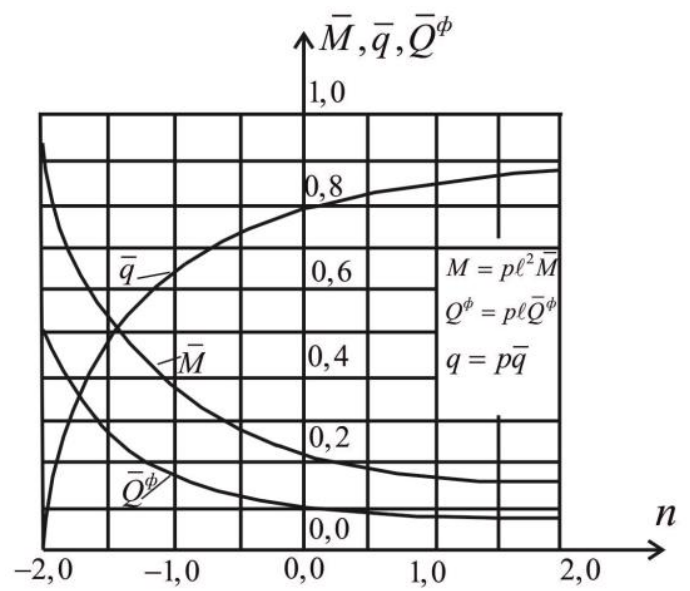

Fig.5. Graphs of changes in reactive pressures and bending of the moment.

At $n=-2$, the distributed foundation pressures under the beam are zero and the load is fully perceived by the concentrated "fictitious" forces. At $n \rightarrow \infty$, the foundation approaches the Winkler one by the nature of work.

\section{Conclusion}

Application of the general variational method of Vlasov-Kantorovich to determine the displacements in the soil layer under conditions of planar deformation allows to take into account heterogeneity of the soil along the thickness of the layer. In this case, it is possible to split the foundation into a multitude of layers with different elastic characteristics and write the solution in matrix form, or use the analytical laws of changing the characteristics.

To design the beam on a single layer foundation with two moduli of subgrade reaction and an elastic modulus that vary with depth in accordance with the power law, an analysis of the variation in the foundation pressure and internal forces in the beam at different intensity changes in the modulus of elasticity is given. With a significant increase in the modulus of elasticity with depth, the calculation results practically coincide with analogous data when using the Winkler scheme.

\section{References}

1. N.M. Atarov, N.N. Leont'ev To a conclusion of differential equations of equilibrium of an elastic layer of soil of variable thickness (MISI Publ., Moscow, 1970)

2. N.M. Atarov, N.N. Leont'ev Izv. VUZov 10 (1971)

3. N.M. Atarov, N.N. Leont'ev On the calculation of beams on the basis of a multilayer (Moscow, 1976) 
4. V.Z. Vlasov, N.N. Leont'ev Beams, plates and shells on elastic base (Fizmatgiz Publ., Moscow, 1960)

5. M.I. Gorbunov-Posadov Calculation of structures on elastic base (Gosstrojizdat Publ, Moscow, 1979)

6. G.K. Klejn Calculation of beams on a continuous basis, continuously inhomogeneous in depth (Gosstrojizdat Publ, Moscow, 1979) 